\title{
ACL2 Meets the GPU: Formalizing a CUDA-based Parallelizable All-Pairs Shortest Path Algorithm in ACL2
}

\author{
David S. Hardin \\ Advanced Technology Center \\ Rockwell Collins \\ Cedar Rapids, IA, USA \\ dshardin@rockwellcollins.com
}

\author{
Samuel S. Hardin \\ Department of Electrical and Computer Engineering \\ Iowa State University \\ Ames, IA, USA \\ sshardin@iastate.edu
}

\begin{abstract}
As Graphics Processing Units (GPUs) have gained in capability and GPU development environments have matured, developers are increasingly turning to the GPU to off-load the main host CPU of numerically-intensive, parallelizable computations. Modern GPUs feature hundreds of cores, and offer programming niceties such as double-precision floating point, and even limited recursion. This shift from CPU to GPU, however, raises the question: how do we know that these new GPU-based algorithms are correct?

In order to explore this new verification frontier, we formalized a parallelizable all-pairs shortest path (APSP) algorithm for weighted graphs, originally coded in NVIDIA's CUDA language, in ACL2. The ACL2 specification is written using a single-threaded object (stobj) and tail recursion, as the stobj/tail recursion combination yields the most straightforward translation from imperative programming languages, as well as efficient, scalable executable specifications within ACL2 itself. The ACL2 version of the APSP algorithm can process millions of vertices and edges with little to no garbage generation, and executes at one-sixth the speed of a host-based version of APSP coded in C - a very respectable result for a theorem prover.

In addition to formalizing the APSP algorithm (which uses Dijkstra's shortest path algorithm at its core), we have also provided capability that the original APSP code lacked, namely shortest path recovery. Path recovery is accomplished using a secondary ACL2 stobj implementing a LIFO stack, which is proven correct. To conclude the experiment, we ported the ACL2 version of the APSP kernels back to $\mathrm{C}$, resulting in a less than $5 \%$ slowdown, and also performed a partial back-port to CUDA, which, surprisingly, yielded a slight performance increase.
\end{abstract}

\section{Introduction}

The computer industry is at a (perhaps temporary) plateau in the race for core speed, but that race has been replaced with a race for more and more cores per die. Graphics Processing Units (GPUs) are at the forefront of this new race - modern GPUs now feature hundreds of cores. As GPUs have gained in capability (GPUs now offer programming niceties such as double-precision floating point, and even limited recursion) and GPU development environments have matured, developers are increasingly turning to the GPU to off-load the main host CPU of numerically-intensive, parallelizable computations. This shift from CPU to GPU, however, raises the question: how do we know that these new GPU-based algorithms are correct?

The question of correctness has several dimensions, including the absence of data races, correctness of the CPU/GPU tools and interfaces, even correctness of the GPU instruction set implementation, all of which are interesting questions, but ones which we will not focus on here. Instead, we wish to focus on issues of basic functional correctness. In this, there is some hope that automated verification tools such as ACL2 will be of some use: GPU kernels are small single-threaded programs, written in a restricted dialect 
of C (e.g., CUDA [10] or OpenCL [6]) and utilizing fixed-size data structures. While this programming model does not match up very well with most verification languages (as noted, for example, in [7]), it is a fair fit to ACL2 using its single-threaded objects, or stobjs [3]. ACL2 is also known for producing relatively speedy and scalable executable specifications, which is valuable for validation of any code that we port from GPU programming languages, such as CUDA or OpenCL, to the ACL2 environment.

Thus, we embarked on the following experiment: we would manually translate a CUDA or OpenCL kernel (or kernels) into ACL2, and see how difficult it was to admit such a kernel into the ACL2 environment, as well as how challenging it would be to do proofs about the translated code. We would then validate the translation by executing the ACL2 version of the kernel, and comparing output with the original. After examining how well the ACL2 version performed by running it on very large data sets, we would "back-port" the ACL2 version to see how well the back-ported version performed. These experiments were performed starting during Christmas break of 2012, and continued on nights and weekends through January 2013.

\section{The ACL2 Theorem Prover}

We utilize the ACL2 theorem proving system [11] for much of our high-assurance verification work, as it best presents a single model for formal analysis and simulation. ACL2 provides a highly automated theorem proving environment for machine-checked formal analysis, and its logic is an applicative subset of Common Lisp [13]. The fact that ACL2 reasons about a real programming language suggests that ACL2 could be an appropriate choice for GPU code verification work. An additional feature of ACL2, single-threaded objects, adds to its strength as a vehicle for reasoning about fixed-size data structures, as will be detailed in the following sections.

\subsection{ACL2 Single-Threaded Objects}

ACL2 enforces restrictions on the declaration and use of specially-declared structures called singlethreaded objects, or stobjs [3]. From the perspective of the ACL2 logic, a stobj is just an ordinary ACL2 object, and can be reasoned about in the usual way. Ordinary ACL2 functions are employed to "access" and "update" stobj fields (defined in terms of the list operators nth and update-nth). However, ACL2 enforces strict syntactic rules on stobjs to ensure that "old" states of a stobj are guaranteed not to exist. This property means that ACL2 can provide destructive implementation for stobjs, allowing stobj operations to execute quickly. In short, an ACL2 single-threaded object combines a functional semantics about which we can readily reason, utilizing ACL2's powerful heuristics, with a relatively high-speed imperative implementation that more closely follows "normal" programming practice.

However, there is no free lunch. As noted by several researchers (e.g. [7]), reasoning about functions on stobjs is more difficult than performing proofs about traditional ACL2 functions on lists which utilize cdr recursion. This difficulty is compounded by the fact that in order to scale to data structures containing millions of elements, all of our functions must be tail-recursive (this would be the case whether we used stobjs or not). It is also well-known in the ACL2 community that tail-recursive functions are more difficult to reason about than their non-tail-recursive counterparts. One of the contributions of this work is a preliminary method to deal with these issues, at least for the case of functions that operate over large stobj arrays. With this method, we have been able to show, for a number of such functions, that a tailrecursive, stobj-based predicate that marches from lower array indices to upper ones is equivalent to a cdr-recursive version of that predicate operating over a simple list. This technique, which we have named 
Hardin's Bridge 1 , relates a traditional imperative loop operating on an array of values to a primitive recursion operating on a list.

\subsection{A Bridge to Primitive Recursion}

As depicated in Figure 1, the process of building this bridge begins by translating an imperative loop, which operates using op on an array dt, into a tail-recursive function (call it $x_{-}$tail) operating on a stobj st containing an array field, also named $d t$. This tail-recursive function is invoked as ( $x$ tail $j$ res st), where res is an accumulator, and the index $j$ counts down from the size of the data array, *SZ*, toward zero. However, the data is indexed as (dti $(-* S Z j)$ st), maintaining the same direction of "march" through the data (i.e., from low to high indices) as the original, imperative loop. x_tail is then shown to be equivalent to a non-tail-recursive function $\mathrm{x}_{-}$prim that also operates over the stobj st. This function is invoked as ( $\mathrm{x}$-prim $\mathrm{k}$ st), where index $\mathrm{k}$ counts up from 0 to $(1-* \mathrm{SZ} *)$. The equivalence of these two functions is established in a manner similar to the defiteration macro found in centaur/misc/iter. lisp in the distributed books, but with a change of variable to adjust the count direction.

We now have a primitive recursion that operates on an array field of a stobj. What we desire, however, is a primitive recursion that operates over lists. One such recursion is as follows:

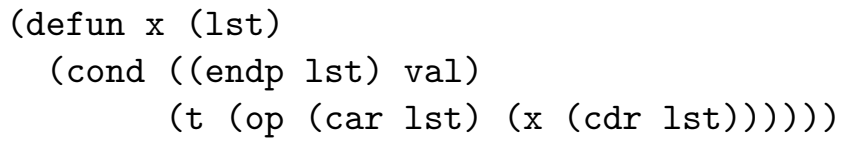

This can be related to x_prim by way of a theorem involving nthcdr:

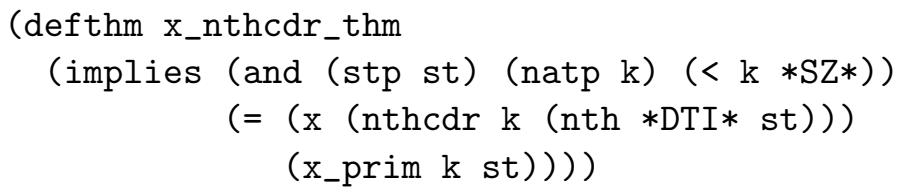

By functional instantiation (setting $\mathrm{k}=0$ ), if the preconditions are met, then

(equal (x (nth $*$ DTI* st)) (x_prim 0 st))

and, by the earlier equivalence,

(equal (x_tail $*$ SZ* 0 st) (x_prim 0 st)

so, finally,

(equal (x_tail $*$ SZ* 0 st) $(x($ nth $*$ DTI $*$ st $)))$

We can now prove theorems about the array-based iteration by reasoning about $\mathrm{x}$, which has a much more convenient form. We have employed this bridge technique on several predicates and mutators, including an array-based insertion sort employing a nested loop.

\footnotetext{
${ }^{1}$ In memory of Scott Hardin, father and grandfather of the two authors: a civil engineer who designed several physical bridges, and a man who valued rigor.
} 

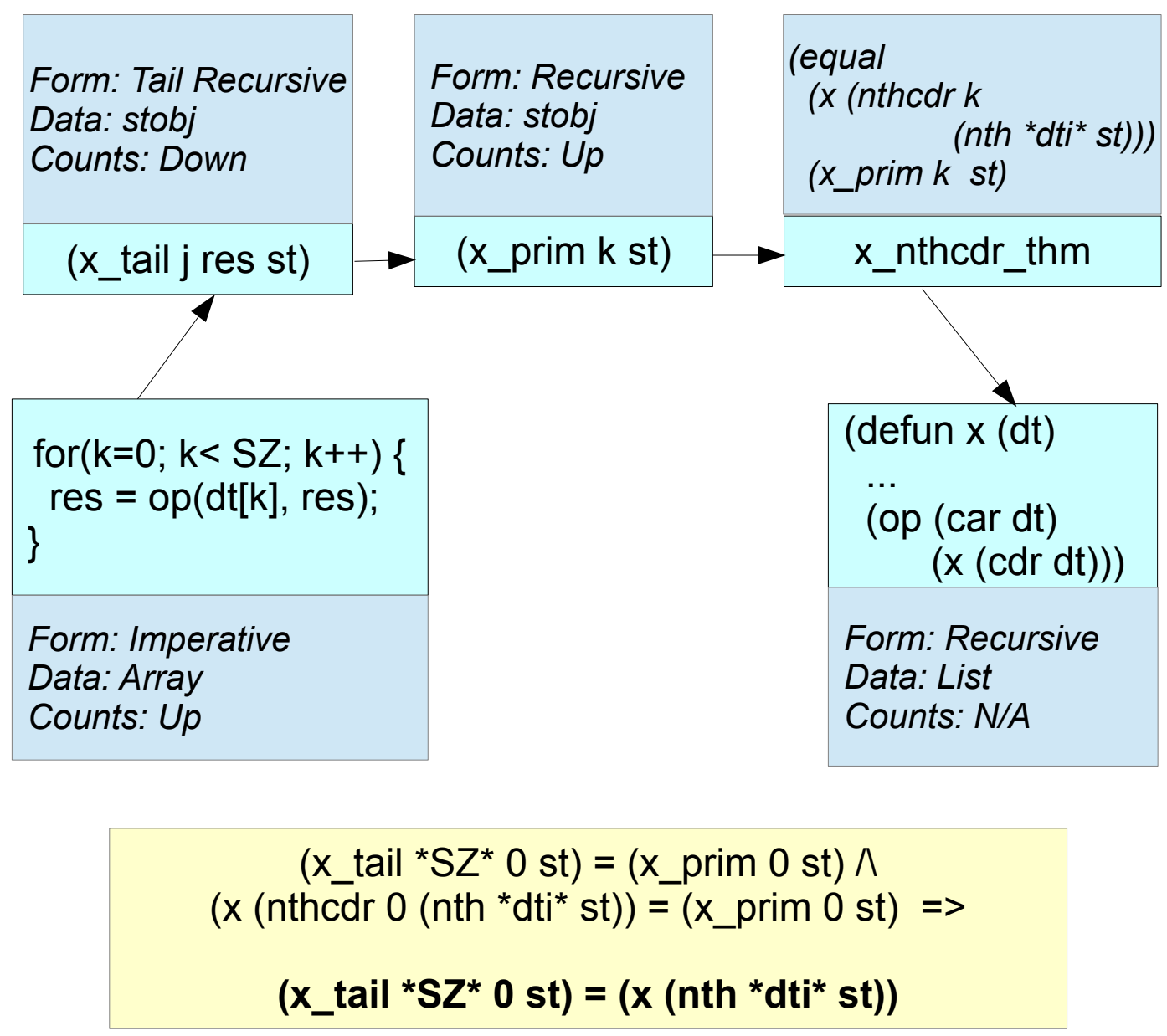

Figure 1: Hardin's Bridge: Relating an imperative loop to a primitive-recursive ACL2 function. 


\section{GPU Programming: CUDA and OpenCL}

Much GPU programming is conducted in either CUDA or OpenCL, although there are many other language alternatives, most of which are bindings to an underlying CUDA or OpenCL system. CUDA is associated with GPUs from NVIDIA; OpenCL supports GPUs from a number of vendors. Both CUDA and OpenCL are a "superset of a subset" of the C family of languages; CUDA supports C++ features, while OpenCL is based on the C99 standard. As one might expect, these languages do not support many of the more powerful (and, one might add, dangerous) features of $\mathrm{C}$, such as function pointers, bit fields, and variable-length arrays and structs - this is actually good news from the formal verification perspective. A bit of not-so-good news is that OpenCL currently does not support recursion, although CUDA does (to a limited degree). Both languages are maturing rapidly, though - double precision floating point is now allowed, for instance, on GPU hardware that supports it.

One of the nice extensions that both of these languages provide is the vector type. (For reasons of space, when discussing language details, we will focus on CUDA; OpenCL has many similar features.) CUDA supports <type >m vector types, where <type > is a scalar type, and $m=1,2,3$, or 4 . Creating and initializing a vector is accomplished as follows:

float4 $f=$ make_float $4(0.0,1.0,2.0,3.0)$;

Accessing a vector component is accomplished by the . [xyzw] convention. For example, the zeroth component of an int 4 variable $j$ containing four integers is $j . x$, and the last component is $j . w$.

The GPU is designed to be a coprocessor to a host CPU. As the GPU comprises many cores, most of the extended language features of CUDA and OpenCL have to do with setup and parallel execution of the cores of this coprocessor. In the CUDA parallel model, any core can theoretically access any memory space; however, there is no guarantee of data coherency. On the other hand, the callers of and execution site for a given function can be restricted. CUDA designates three function type qualifiers: _-device -- $_{\text {, }}$

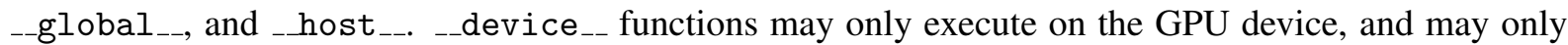
be called by other functions on the device. _-global__ declares a function as being a kernel function, which executes on the GPU device, but is callable from the host only. Finally, _host_- functions, as the name implies, execute on the host, and are callable from the host only.

The CUDA hardware model consists of a number of multiprocessors, which execute asynchronously in parallel. Each multiprocessor consists of a number of stream processors, or more commonly, cores. Each kernel is dispatched by the host CPU onto a multiprocessor, where it is divided into groups of threads. Each thread is part of a block, and each block is one element of a grid. All threads in a grid execute in Single Instruction, Multiple Data (SIMD) fashion. Each thread within a block and each block within a grid are given unique identifiers that can be referenced in the developer's CUDA source code. Note that in order to take advantage of potential data parallelism, the developer must write her code in a particular way, e.g. array indices need to be stratified by block ID and thread ID, as in:

int tid $=$ blockIdx.x $*$ MAX_THREADS_PER_BLOCK + threadIdx.x;

\section{The GPU Example: All-Pairs Shortest Path}

In scanning the GPU literature for examples, we looked for implementations of algorithms that would be familiar to a theorem proving audience, and that primarily involved integer operations. After a bit of searching, we found an interesting example in a CUDA implementation of an all-pairs shortest path (APSP) algorithm for weighted graphs documented by Harish and Narayanan [8]. The algorithm was 

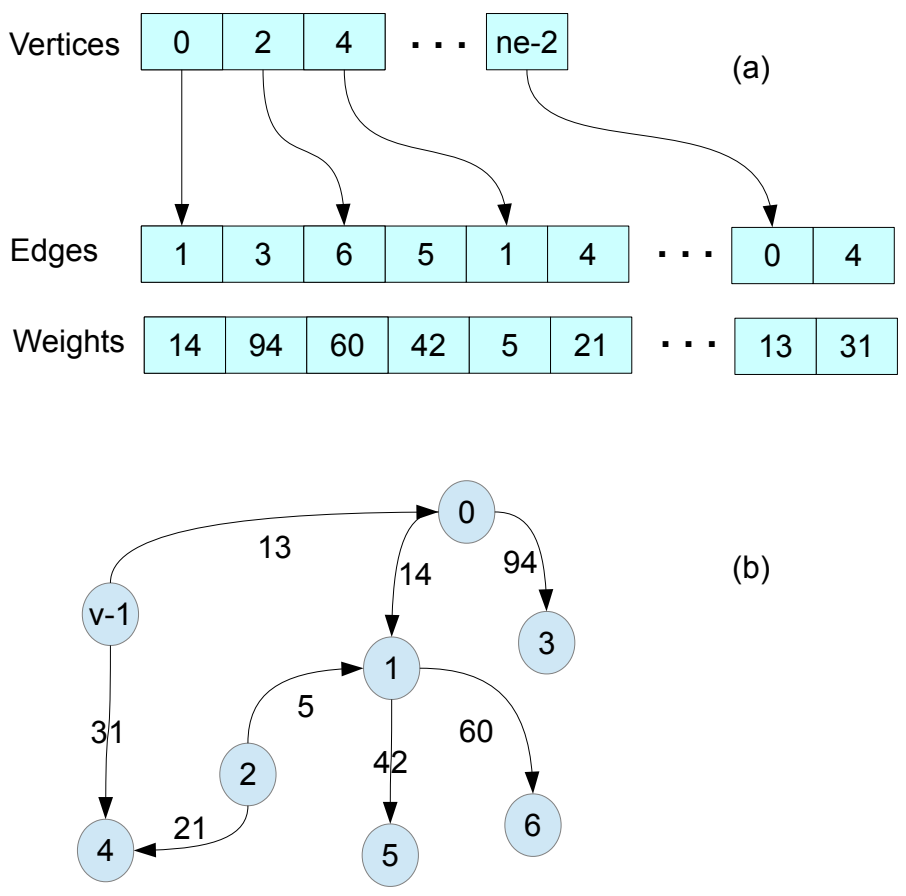

(b)

Figure 2: (a) Vertices, Edges, and Weight Arrays. (b) Graph fragment from the data in (a).

later translated to OpenCL by Ginsburg [4]. Notably, Ginsburg found an algorithmic error in Harish and Narayanan's original paper, so we were keen to explore this issue as well.

The APSP algorithm proceeds by repeated application of Dijkstra's single-source shortest path (SSSP) algorithm, applied to a data structure in which the graph vertices, edges, weights, and computed costs are encoded as arrays. The vertex array contains indices into the edge array, whereas the edge array contains vertex indices, as shown in Figure 2. The weight array contains the weight of each edge, and thus is the same size as the edge array. The cost array contains the accumulated weight of the shortest path from a given source vertex to each destination vertex, as computed by Dijkstra's algorithm. The costs for the individual shortest path runs are copied into a final result array of size $*$ MAX_VERTICES $* *$ $*$ MAX_SOURCES*. For a full application of APSP, that size would be the square of the number of vertices. For large graphs, a full APSP would exceed the memory capacity of current personal computers, and would take quite a long time even if the memory were available. Thus the number of source vertices is usually set to be quite a bit less than the number of vertices. (n.b.: If full APSP were desired for a graph of a million vertices, for example, one could perform the algorithm on, say, 4000 source vertices at a time, and record the shortest path cost data to disk. Fortunately, this is quite a rare use case.)

One will immediately note a deficiency in this data structure: nowhere are the shortest paths themselves stored, just their costs. Apparently, this is common for APSP algorithms, no doubt due to space considerations, and also due to the fact that most shortest paths are not "interesting" to the analyst. Fortunately, once one has the shortest path cost from vertex A to B, it is relatively easy to recover the shortest path from A to B. We have written an ACL2 function to perform path recovery, using an ACL2 stobj that 
implements a LIFO stack.

\subsection{Harish's Algorithm}

Harish and Narayanan [8] present a pseudocode overview of their APSP implementation in their Algorithm 7. We reproduce that algorithm, which we will refer to from now on as Harish's algorithm, below:

Copy vertex array $V_{a}$, edge array $E_{a}$, and weight array $W_{a}$ from $\mathrm{G}(\mathrm{V}, \mathrm{E}, \mathrm{W})$

Create mask array $M_{a}$, cost array $C_{a}$, and updating cost array $U_{a}$ of size $\mathrm{V}$

for $S$ from 1 to $V$ do

$M_{a}[\mathrm{~S}] \leftarrow$ true

$C_{a}[\mathrm{~S}] \leftarrow 0$

while $M_{a}$ not Empty do

for each vertex $\mathrm{V}$ in parallel do

Invoke CUDA_SSSP_KERNEL1 $\left(V_{a}, E_{a}, W_{a}, M_{a}, C_{a}, U_{a}\right)$ on the grid

Invoke CUDA_SSSP_KERNEL2 $\left(V_{a}, E_{a}, W_{a}, M_{a}, C_{a}, U_{a}\right)$ on the grid

end for

end while

end for

Harish and Narayanan observe that for large graphs and modern CPU/GPU architectures, this algorithm performs better than previously developed parallel APSP algorithms, such as the parallel Floyd Warshall APSP algorithm - the intermediate results are only of size $O(V)$, and the algorithm involves an $O(V)$ operation looping over $O(V)$ threads, as opposed to an $O(V)$ operation looping over $O\left(V^{2}\right)$ threads [8].

In Harish's algorithm, the two SSSP kernels proceed as follows in each major iteration (that is, while the mask array is not all false). The first SSSP kernel checks whether the mask entry for each vertex is set. If the mask for a given vertex is set, it fetches its current cost from the cost array, as well as the neighboring edge weights from the weight array. The cost of each neighbor vertex is updated if its current cost is greater than the cost of the current vertex plus the weight of the edge from the original vertex to the neighbor vertex. This updated cost is recorded not in the cost array, but in a secondary updating cost array, due to the lack of memory coherence in the CUDA programming model. The second kernel then updates each element of the cost array to the value in the updating cost array value if the updating cost array value is less, and also sets the corresponding mask array value so that the algorithm will examine that vertex on a future iteration for further possible cost reductions. Otherwise, the updating cost array element is set to the value of the corresponding cost array element.

To get a feel for what the kernels look like, let's examine the first kernel of the APSP algorithm (Figure 3), courtesy of Harish [9] (with a few cosmetic changes added by the authors).

As noted above, the basic index (tid) is expressed in terms of the block ID as well as the thread ID within the block; this allows the CUDA compiler to distribute the data parallel processing to the cores in a grid. The other parallel programming wrinkle apparent in this code is the use of the atomicMin function, which as its name implies, atomically updates the value pointed to by its first argument with the minimum of the value pointed to by the first argument and the second argument. 


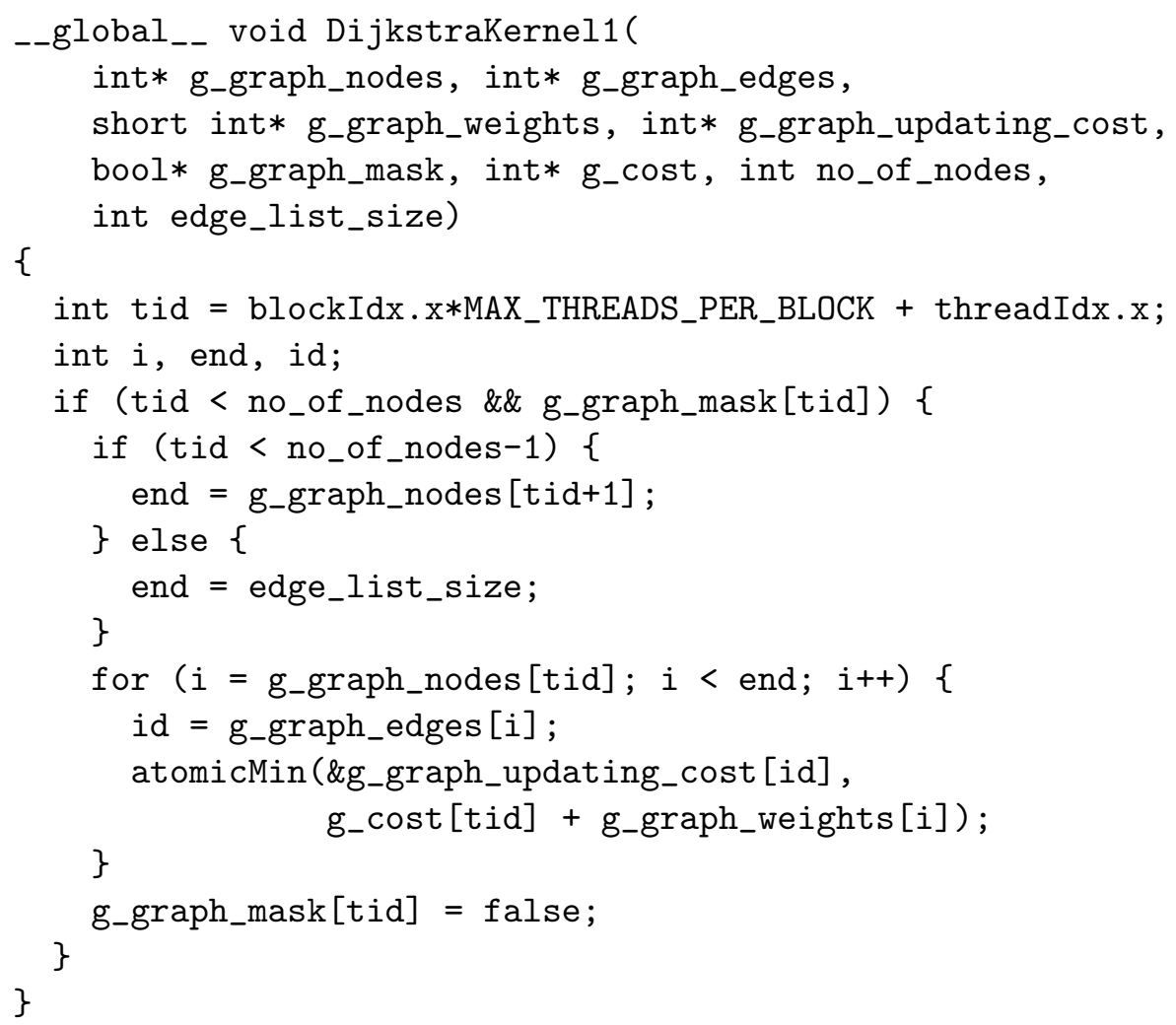

Figure 3: A Single-Source Shortest Path Kernel in CUDA. 


\section{$5 \quad$ APSP in ACL2}

Our translation of Harish's algorithm into ACL2 was aided by the existence of Ginsburg's OpenCL port [4]. Not only did Ginsburg point out a bug in the presentation of CUDA_SSSP_Kernel1, but he also provided, in the source code accompanying his book chapter, a non-parallel reference implementation in C, which was quite helpful. So, following Ginsburg's lead, we also decided to develop a non-parallel reference implementation, this time in ACL2. We could possibly have explored the use of ACL2(p) [16] in our modeling, and developed a more faithful parallel implementation, but were uncertain about the interplay of ACL2(p) and stobjs. (One of the reviewers of this paper noted that, indeed, plet and stobjs are incompatible.)

The APSP implementation in ACL2 is too lengthy to present in a paper, but the sources will be made available. We begin our brief tour of the ACL2 code with a presentation of the basic stobj used by all functions in the APSP implementation.

\subsection{Graph Definitions in ACL2}

The basic single-threaded object declaration for a weighted graph of 1 million vertices and 10 edges per vertex is as follows:

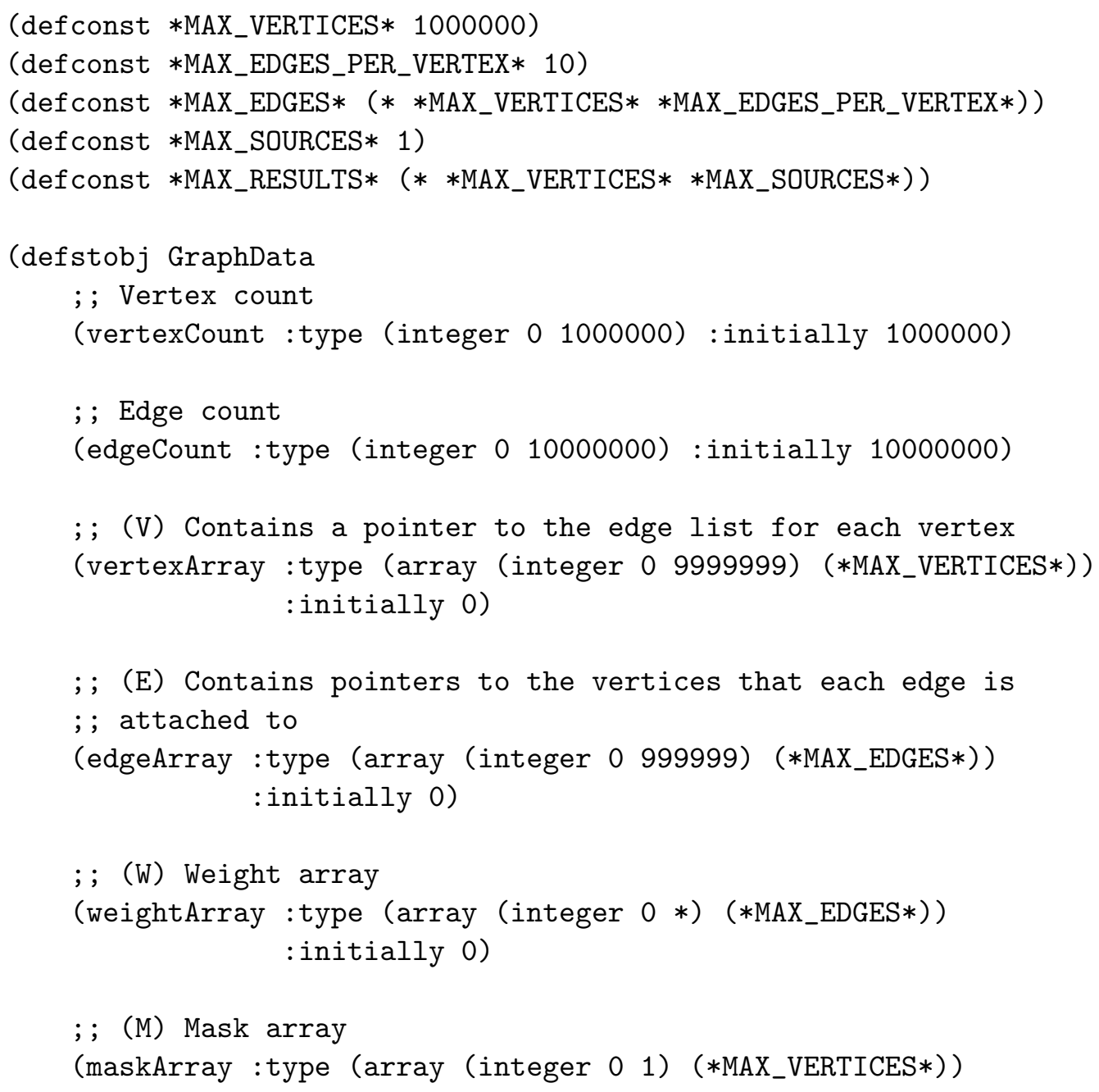




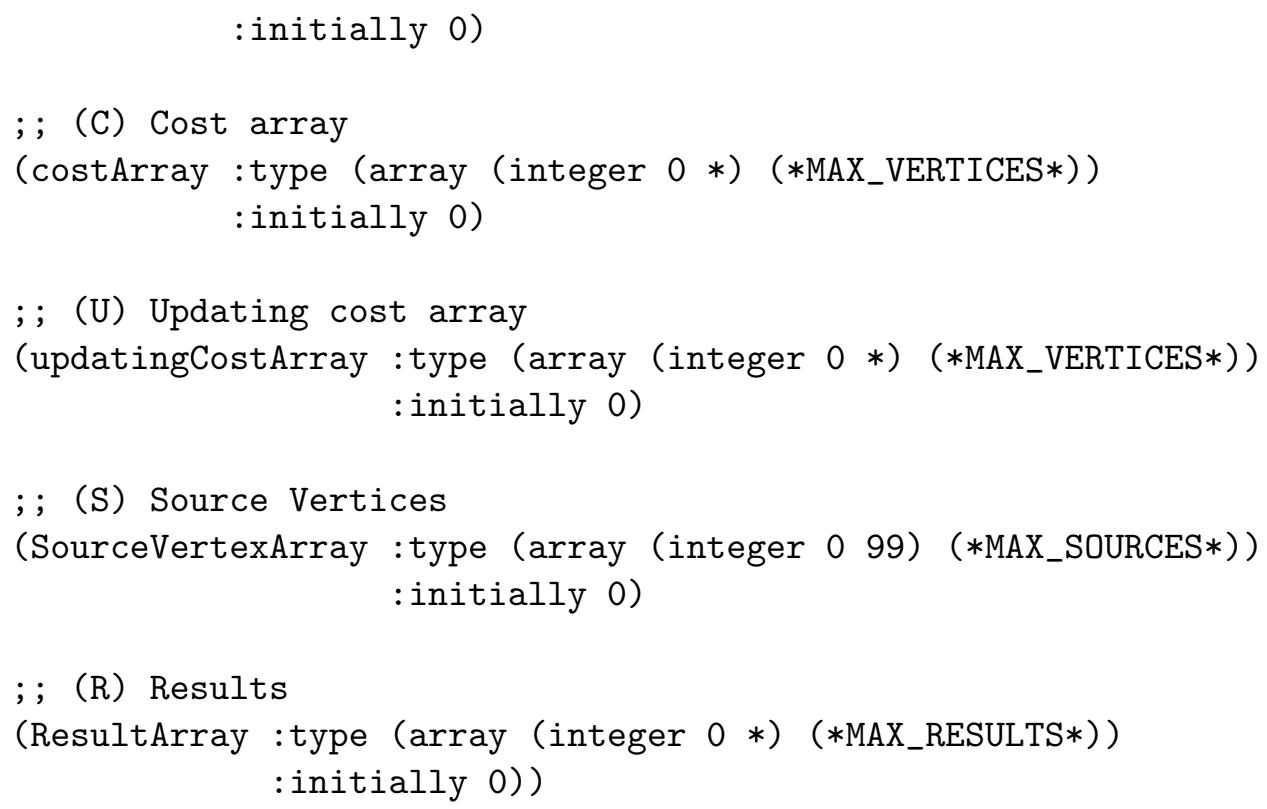

First note that the algorithm is fundamentally parameterized by the constants $*$ MAX_VERTICES*, $*$ MAX_EDGES_PER_VERTEX $*$, and $*$ MAX_SOURCES $*$. The latter can be adjusted up to $*$ MAX_VERTICES $*$, in the case of a true all-pairs shortest path computation. Next, observe that first two elements of the stobj, vertexCount and edgeCount, are not strictly necessary. But, they were present in Ginsburg's OpenCL and (non-parallel) $\mathrm{C}$ reference versions, so we duplicate them here. The presence of these two elements at the beginning of the stobj also stops ACL2 from rewriting, e.g. (nth *EDGEARRAYI* GraphData) into (cadr GraphData) during proofs, which can be annoying to deal with. Also observe that we are using arbitrary precision integers for most data here. Happily, we have been able to proceed at reasonable speed without resorting to, e.g. 64-bit integers. And remarkably, due to the limited arithmetic involved, we can do significant runs over large graphs and allocate less than 2000 bytes of heap data (according to time\$). Finally, one may have thought it more elegant to declare the maskArray elements to be of type (or $t \mathrm{nil}$ ). This turned out to be both harder to reason about (ACL2 has much better support for integer-listp), and significantly slower to execute, so we abandoned it.

\subsection{ACL2 Translation Techniques}

Once the main data structure was in place, we recoded all loops as tail-recursive functions, accepting the GraphData stobj as a parameter, and returning it if any of its fields were updated. Termination proofs were not difficult in general; only one function required a dummy "countdown" parameter to help with termination analysis.

One issue that we had in the translation was the treatment of the various for loops. In ACL2, it is advantageous to code in a "decrementing" style; however, in most imperative coding cultures, a for loop that increments an index through an array is the preferred idiom. We were somewhat concerned that by changing the direction from incrementing to decrementing, that we might accidentally "break" the code by, e.g. failing to notice some data dependency of "later" elements of an array on "earlier" elements. The solution we arrived at was to have a decrementing index (call it ix), but to address the arrays in question, as, for example, (vertexArrayi (- *MAX_VERTICES* ix) GraphData).

The result, while somewhat verbose (n.b.: the verbosity could be hidden with a simple macro), allows the ACL2 code to "march" through the array from low indices to high indices as in the original, 


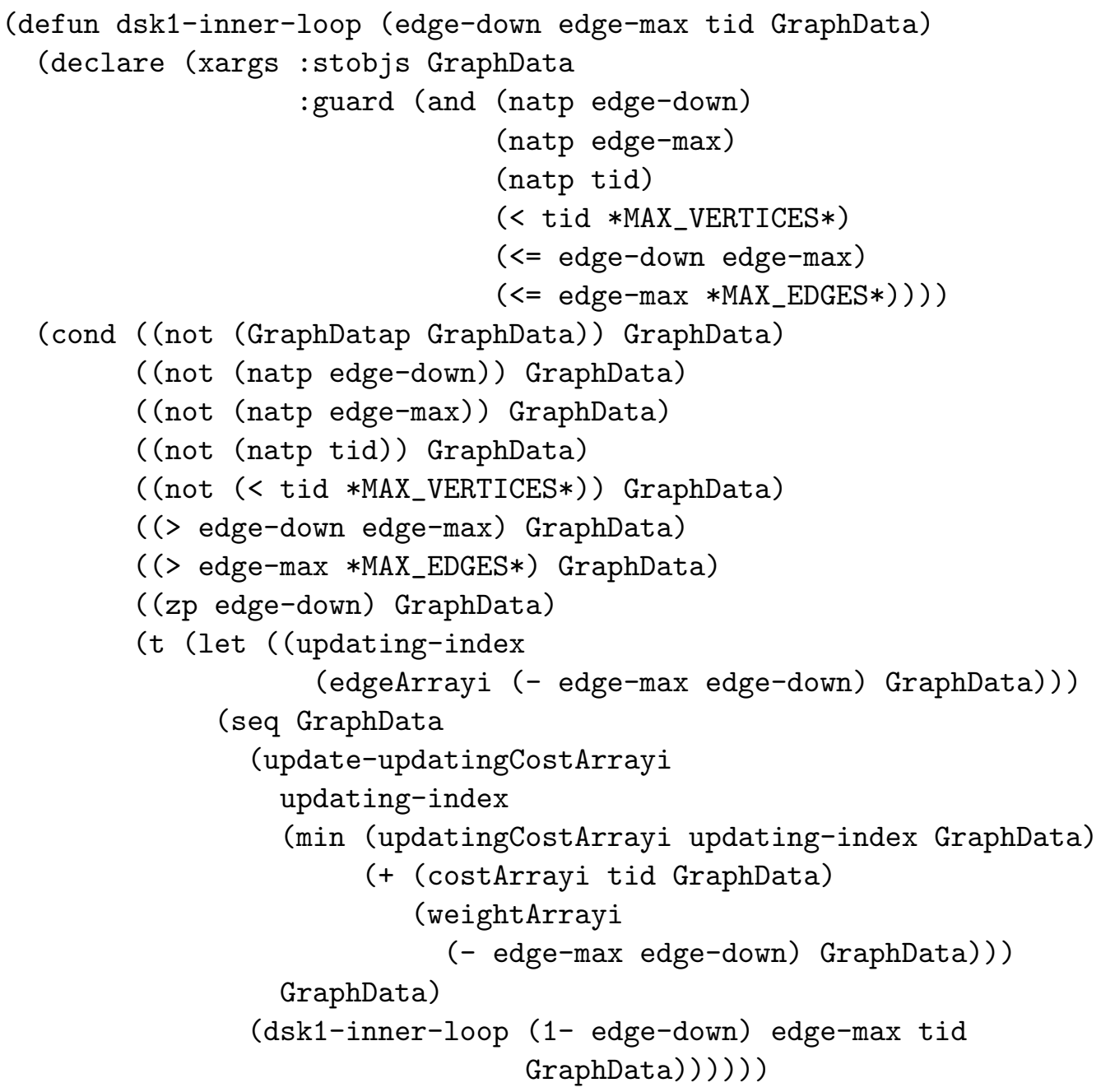

Figure 4: Single-Source Shortest Path Kernel inner loop in ACL2.

while maintaining a decrementing loop counter parameter that ACL2 prefers (and whose initial value, conveniently, would be something simple like $*$ MAX_VERTICES $*$ ), and also allowing a nice (zp ix) test to stop the loop. So, let us look at the ACL2 translation of the inner loop of Figure 3, shown in Figure 4. The first dozen lines or so are just necessary guard conditions, and the corresponding checks in the body of the ACL2 function. From there, it's a fairly direct translation from the CUDA inner loop to a tail-recursive function, with J Moore's seq macro eliminating some of the let binding clutter.

The entirety of the translation, including all the guard proofs and related theorems, as well as the occasional comment, comes to nearly 2600 lines of ACL2 code. Some of that code, however, provides functionality not found in the original CUDA code, namely shortest path recovery. Some 160 lines of that additional code constitutes a stobj-based implementation of a LIFO stack (and its basic correctness proofs) that was needed for the shortest path recovery implementation. 


\subsection{Lessons Learned from the Translation}

The translation to ACL2 revealed several flaws in the original code. First, as is the case with most C-based code, plain int types were used when some variant of unsigned types could have been used instead. Since all of the GraphData components are natural numbers, and all operations on the GraphData are either additions or comparisons, it would have been smarter to use unsigned types. Additionally, no particular care was taken to guard against array index overflows, another common problem with C-based code. The formalization in ACL2 demonstrates that if the developers had spent just a little extra time in declaring and guarding the array indices, the algorithm could be shown to safely stay within its various array bounds.

Kernel 1, as originally written, also suffers from a possible integer overflow error in the computation of the updating cost array - the addition of the cost array element and the weight element could wrap around. The wrapped-around value could then propagate to the updated cost array, as the cost is suddenly much lower. This, in turn, could lead to an inaccurate shortest path computation. Ginsburg's code [4] ameliorates this issue by changing the cost, weight, and updating cost arrays to use floating point; but this has a significant impact on performance. Harish's code [9] makes wrap-around somewhat less likely by declaring the weight array to be a short (16-bit) integer; nonetheless, in the worst case one could still have wrap-around of a signed 32-bit cost after 65,539 additions, which is certainly possibly for shortest path searches through large graphs. One could remedy this issue by changing the min computation in Kernel 1 to something like

cwsum $=C[i]+W[j]$

$\min (\mathrm{U}[\mathrm{k}]$, (cwsum < C [i])?WT_MAX:cwsum);

where WT_MAX is the largest possible weight value. Preliminary results indicate that the addition of such a wrap-around check has negligible impact on the algorithm's runtime for large graphs.

The final flaw to be discussed is the error in Kernel 1 disclosed by Ginsburg. The problem in the Harish and Narayanan paper [8] (a problem which is fixed in the source code retrieved from Harish's web site [9]) was that the weight array was being indexed by an improper value; in essence, it was being indexed by an element of the edge array, $\mathrm{E}[\mathrm{i}]$ instead of just $\mathrm{i}$. One can see the problem by noting that weight array indices should be in the range $0 \ldots *$ MAX_EDGES $*-1$, while the edge array, being an array of vertex indices, should have values in the range $0 \ldots *$ MAX_VERTICES $*-1$, usually a much smaller number. When this error is introduced into the ACL2 code, the guard proofs produce a subgoal (which it fails to prove without further assistance) whose conclusion is basically E[i] < *MAX_EDGES*. An analyst with some knowledge of the data structure would spot this as a strange subgoal to generate (as opposed to $E[i]<*$ MAX_VERTICES*). However, this subgoal can be proven eventually, so it can't be said that ACL2 found the error. The only way for ACL2 to uncover the flaw would be to attempt an equivalence proof between Kernel 1 and an existing version of Dijkstra's algorithm specified in ACL2, a task which remains as future work.

\subsection{Guards and Performance}

Given that we wished to validate the ACL2 implementation of APSP utilizing large graphs (1 million vertices and 10 million edges), we needed a reasonably efficient executable specification. In addition to exploiting tail recursion and the in-place update capability that stobjs provide, we also knew that we needed to verify the guards of all performance-relevant functions in order to achieve acceptable results. At first, this seemed a daunting task; but it turned out to be almost routine after a while. Many of the 
guard conjectures we needed to prove for non-leaf functions stated that if that function called another function foo with a GraphDatap argument, foo would return a GraphDatap result. This, in turn, could be broken down into a series of proofs that the "types" of the components of the GraphData stobj were preserved across a call of foo (by "type", we mean a basic predicate such as natp). Happily, most GraphData components were unaffected by a given function, and proving that fact was particularly easy. For the other components that were updated by function foo, ACL2 usually had little difficulty in establishing that the updates preserved that component's "type". In the end, having verified guards reduced the execution time of our tests by $40 \%$.

One interesting property that arose during guard proof development was the need to establish that the vertexArray elements were nondecreasing. (This happens to be the case, given how the vertex array is initialized by the init-vertex-array function.) Thus, a number of functions ended up with an additional (vertices-nondecreasing GraphData) guard condition. Note that we reason about this predicate using the bridge technique described in Section 2.2 .

\subsection{Performance Results}

The ACL2 version of Harish's algorithm can find the shortest path from a given source vertex to each of a million destination vertices, following 10 million edges in less than 8 seconds on a late 2012 MacBook Pro with a $2.5 \mathrm{GHz}$ Intel Core i5 dual-core CPU, and with little to no garbage generation. The performance of the algorithm is linear in the number of source vertices, at least up to the 100 source vertices tested so far (for a one million vertex graph with 10 edges per vertex).

The performance of the ACL2 version of Harish's algorithm relative to Ginsburg's non-parallel C reference implementation is shown as a function of the number of source vertices in Figure 5. The performance of both is linear in the number of source vertices, and the ACL2 version consistently executes at one-sixth the speed of the $\mathrm{C}$ reference implementation - a very respectable result for a theorem prover, utilizing arbitrary-precision arithmetic. As a final comparison, Harish and Narayanan report that their GPU-based implementation is some 70 times faster than the CPU-only version [8].

\section{Back-porting to $\mathrm{C}$ and CUDA}

To conclude our experiment, we ported the ACL2 version of the APSP kernels back to C and CUDA, to see what effect the tail-recursive style would have on performance. We were particularly interested to see if the CUDA compiler (actually a complicated frontend to GCC) would truly support tail recursion, and whether the $\mathrm{C}$ version would suffer in performance from moving inner loops into their own functions. The methodology used here was simple, and purely empirical - keep increasing the optimization level on the compiler until performance stopped improving or the generated code became broken. We found that for $\mathrm{C}$ (using clang), performance of the tail-recursive extracted inner loops was much poorer than the baseline $\mathrm{C}$ reference implementation at low optimization levels, but that higher optimization (-03) improved matters significantly, with the tail-recursive version showing only a $3 \%$ slowdown.

In order to obtain results for the back-port of the tail-recursive ACL2 formulation to CUDA, we first updated Harish's code [9] to allow it to compile and run in the current CUDA environment (CUDA 5.0), which we downloaded from the CUDA website [10] onto a late 2012 iMac equipped with an NVIDIA GeForce GT 650M GPU with 512MB of memory. For our initial CUDA back-port, we replaced only the inner loop of CUDA_SSSP_KERNEL1 (see Figure 3) with a tail-recursive function hand-translated back from the ACL2 code of Figure 4. We conducted complete APSP runs (i.e., we computed the shortest 


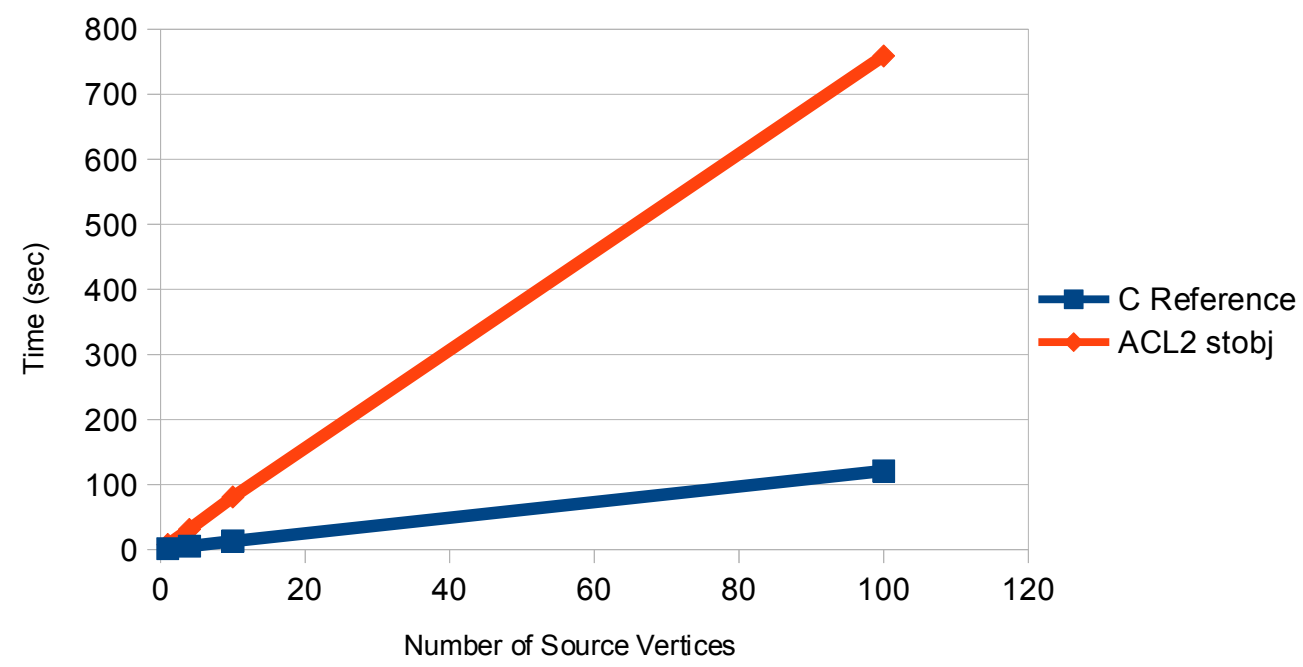

Figure 5: Time to find all shortest paths from a number of source vertices.

paths between all pairs of vertices) for a randomly generated graph of 100,000 vertices and 5 edges per vertex using both Harish's non-recursive CUDA code as well as our tail-recursive alternative. We timed our results using (updated) timing code already present in Harish's sources, and compared the output shortest path costs to ensure that the non-recursive and tail-recursive versions both yielded the same results. Surprisingly, our tail-recursive back-port from ACL2 was slightly faster than Harish's non-recursive version, but only by $1.8 \%$.

\section{Related Work}

The use of ACL2 single-threaded objects to speed graph search problems enjoys a long history. Wilding [17] was the first, in 2000, to use a stobj to speed an ACL2 algorithm, originally documented by Moore [14], for finding a path between two given vertices in an unweighted graph. Like our work, Wilding uses a vertex array and a mask array (perhaps more descriptively, Wilding calls this array a mark array). Unlike our representation, Wilding's vertex array elements are lists of children of that vertex. Greve and Wilding followed this work in 2003 [5] with a version that utilized MBE to justify a major optimization used in the 2000 paper.

GPU programming is a relatively new activity, so there has not been much research into the verification of GPU programs, toolchains, or hardware. Two notable tools are PUG [12] and GPUVerify [2]. PUG is a data race analyzer for CUDA programs, utilizing an SMT solver. GPUVerify translates OpenCL and CUDA kernels into Boogie [1] in order to find data races. Neither tool focuses on basic functional correctness. 


\section{Conclusion and Future Work}

We have formalized a parallelizable all-pairs shortest path (APSP) algorithm for weighted graphs, originally coded in NVIDIA's CUDA language, in ACL2. The ACL2 specification is written using a singlethreaded object (stobj) in tail recursive style. The ACL2 version of the APSP algorithm scales to millions of vertices and edges with little to no garbage generation, and executes at one-sixth the speed of a hostbased version of APSP coded in C. We also provided capability that the original APSP code lacked, namely shortest path recovery. Path recovery is accomplished using a secondary ACL2 stobj implementing a LIFO stack, which was proven correct. To bring our work back to where it began, we ported the ACL2 version of the APSP kernels back to C, where we observed a mere 3\% slowdown, and also performed a partial back-port to CUDA, where we measured a slight performance increase of $1.8 \%$.

Future work should focus on using ACL2 to verify that Harish's algorithm does indeed implement APSP. This will involve creating an ACL2 formalization of APSP, building upon an existing formalization of Dijkstra's shortest path algorithm by Moore and Zhang [15]. Work should also continue on improving techniques for reasoning about stobjs; some progress has been made during the current effort, but much more work is needed in order to make the process easier.

Additional future work on the APSP algorithm implementation may involve setting an upper bound on the values of elements of the cost, weight, and updating cost arrays; as well as investigating the use of unsigned-bytep types for all arrays. In the general area of GPU verification, many opportunities present themselves, including hardware and toolchain verification, as well as verification of the CPU/GPU interface.

\section{Acknowledgments}

We thank Dave Greve and Konrad Slind for their sage advice, as well as the anonymous reviewers for their thorough and thoughtful comments.

\section{References}

[1] M. Barnett, B. Chang, R. Deline, B. Jacobs \& K.R.M. Leino (2005): Boogie: A Modular Reusable Verifier for Object-Oriented Programs. In: FMCO 2005, doi:10.1007/11804192_17.

[2] Adam Betts, Nathan Chong, Alastair Donaldson, Shaz Qadeer \& Paul Thomson (2012): GPUVerify: A Verifier for GPU Kernels. In: Proceedings of OOPSLA 2012, ACM, doi:10.1145/2384616.2384625.

[3] Robert S. Boyer \& J Strother Moore (2002): Single-Threaded Objects in ACL2. PADL 2002, doi:10.1007/3540-45587-6_3.

[4] Dan Ginsburg (2011): Parallelizing Dijkstra's Single-Source Shortest-Path Graph Algorithm. In Aaftab Munshi, Benedict Gaster, Timothy Mattson, James Fung \& Dan Ginsburg, editors: OpenCL Programming Guide, Addison-Wesley Professional, pp. 411-424.

[5] David Greve \& Matthew Wilding (2003): Using MBE to Speed a Verified Graph Pathfinder. In: ACL2 Workshop 2003.

[6] Khronos Group: OpenCL - The Open Standard for Parallel Programming of Heterogeneous Systems. Available at http://www.khronos.org/opencl

[7] David S. Hardin \& Samuel S. Hardin (2009): Efficient, Formally Verifiable Data Structures using ACL2 Single-Threaded Objects for High-Assurance Systems. In S. Ray \& D. Russinoff, editors: Proceedings of the Eighth International Workshop on the ACL2 Theorem Prover and its Applications, ACM, pp. 100 - 105, doi: $10.1145 / 1637837.1637853$ 
[8] Parwan Harish \& P.J. Narayanan (2007): Accelerating Large Graph Algorithms on the GPU using CUDA. In: IEEE High Performance Computing - HiPC 2007, Lecture Notes in Computer Science 4873, Springer, pp. 197-208, doi:10.1007/978-3-540-77220-0_21.

[9] Pawan Harish: Graph Algorithms on CUDA (2007-2011). Available at http://researchweb.iiit.ac. in/ harishpk.

[10] NVIDIA Inc.: CUDA Parallel Programming and Computing Platform. Available at http://www.nvidia. com/object/cuda/home_new.html

[11] Matt Kaufmann, Panagiotis Manolios \& J Strother Moore (2000): Computer-Aided Reasoning: An Approach. Kluwer Academic Publishers, doi 10.1007/978-1-4757-3188-0.

[12] G. Li \& G. Gopalakrishnan (2010): Scalable SMT-based verification of GPU Kernel Functions. In: FSE 2010, doi: $10.1145 / 1882291.1882320$

[13] LispWorks Ltd.: Common Lisp HyperSpec. Available at/http://www.lispworks . com/documentation/ HyperSpec/Front/index.htm

[14] J Strother Moore (2000): An Exercise in Graph Theory. In: Computer-Aided Reasoning: ACL2 Case Studies, Kluwer Academic Publishers, doi $10.1007 / 978-1-4757-3188-0 \_5$.

[15] J Strother Moore \& Q. Zhang (2005): Proof Pearl: Dijkstra's Shortest Path Algorithm Verified with ACL2. In J. Hurd \& T. Melham, editors: 18th International Conference on Theorem Proving in Higher Order Logics: TPHOLs 2005, Lecture Notes in Computer Science 3603, Springer, pp. 373-384, doi:10.1007/11541868_24.

[16] David L. Rager (2006): Adding parallelism capabilities to ACL2. In: Proceedings of the sixth international workshop on the ACL2 theorem prover and its applications, ACL2 '06, ACM, New York, NY, USA, pp. 90-94, doi: $10.1145 / 1217975.1217994$.

[17] Matthew Wilding (2000): Using a Single-Threaded Object to Speed a Verified Graph Pathfinder. In: ACL2 Workshop 2000. Available as University of Texas Dept. of CS TR 00-29. 